\title{
The contributions of Steve Lawn to the science, advocacy and policy of HIV-associated TB
}

IT IS WITH GREAT SADNESS that we learned about the death of Steve Lawn. To commemorate his groundbreaking and influential work on human immunodeficiency virus (HIV) associated tuberculosis (TB), we aim to highlight several of his most notable contributions to research and advocacy.

Professor Lawn will be remembered for his trailblazing work on improving the diagnosis of HIV-associated TB. Given the irreversible cost of delayed diagnosis of TB in PLHIV [people living with HIV]', ${ }^{1}$ he argued for 'stretching diagnostics to their limits' 2 and refused to accept the status quo of 'outdated technologies that are blunt and ineffective tools' for the diagnosis of TB in PLHIV. ${ }^{3}$ Through rigorous studies, he evaluated current and new diagnostics for their accuracy and impact on important clinical outcomes among these most vulnerable patients. Based on sound evidence, he supported the systematic use of culture, ${ }^{4}$ and later Xpert ${ }^{\circledR}$ MTB/ RIF, ${ }^{5}$ in the screening for TB in PLHIV eligible for antiretroviral treatment (ART), even among those without classical TB symptoms. He was also a tenacious advocate for the use of lipoarabinomannan (LAM) for the diagnosis of TB in PLHIV, publishing 20 papers describing the underlying pathophysiology and documenting the clinical usefulness of LAMbased tests. His work on LAM started with the evaluation of a serum test in Ghana in $1997,{ }^{6}$ evolved to descriptive studies $^{7,8}$ and a still ongoing clinical trial, ${ }^{9}$ and culminated in the World Health Organization policy guidance for use of lateral flow urine LAM for the diagnosis and screening of active TB in PLHIV. While recent promising developments in TB diagnostics may have eroded the sense of urgency to do more, Prof Lawn provided a steadfast voice in support of the importance of continued investment in the developmental pipeline for rapid, accurate, affordable and implementable point-of-care diagnostic tests. ${ }^{10}$

Prof Lawn was deeply committed to the prevention of the hundreds of thousands of HIV-associated TB deaths occurring each year in sub-Saharan Africa, and used his influential position to challenge the lack of urgency in the global response to this crisis. He asked pointedly 'when will we act?', 11 and wrote that 'the need for action was no less urgent now [in 2014] than it was 25 years ago'. 12 Prof Lawn was one of the first to highlight that the DOTS strategy alone was insufficient to control the HIV-associated TB epidemic. ${ }^{13,14}$ By wondering 'will ART do it?', he advanced the debate on the role of ART in controlling the TB-
HIV epidemic in sub-Saharan Africa, and advocated to 'shut the stable door early' through implementation of treatment as prevention and test and treat strategies. ${ }^{15}$

We will miss Steve's intelligence, scientific integrity, passion for public health, and vision for a better life for those living with HIV and TB. His mission to improve the public health response to HIV-associated TB remains an unmet goal. We believe that Steve's work and tenacity serves as a template for how we can advance both science and policy in support of a brighter future.

AnNelies Van Rie, MD, MPH*

Ted Cohen, MD, $\mathrm{MPH}^{\dagger}$

*Department of Epidemiology and Social Medicine University of Antwerp Antwerp, Belgium

${ }^{\dagger}$ Department of Epidemiology of Microbial Diseases Yale School of Public Health

New Haven, CT, USA e-mail: Annelies.VanRie@uantwerpen.be

\section{References}

1 Lawn S D, Griffin G E. The irreversible cost of delayed diagnosis of tuberculosis in HIV co-infected persons in sub-Saharan Africa. Int J Tuberc Lung Dis 2001; 5: 200-203.

2 Lawn S D, Wood R. Tuberculosis screening in patients starting antiretroviral therapy in sub-Saharan Africa: stretching diagnostics to the limits. Clin Infect Dis 2011; 52: 276-277.

3 Lawn S D. Advances in diagnostic assays for tuberculosis. Cold Spring Harb Perspect Med 2015; 5(12).

4 Lawn S D, Kranzer K, Edwards D J, McNally M, Bekker L-G, Wood R. Tuberculosis during the first year of antiretroviral therapy in a South African cohort using an intensive pretreatment screening strategy. AIDS 2010; 24: 1323-1328.

5 Lawn S D, Brooks S V, Kranzer K, et al. Screening for HIV-associated tuberculosis and rifampicin resistance before antiretroviral therapy using the Xpert MTB/RIF assay: a prospective study. PLOS MED 2011; 8: e1001067.

6 Lawn S D, Frimpong E H, Nyarko E. Evaluation of a commercial immunodiagnostic kit incorporating lipoarabinomannan in the serodiagnosis of pulmonary tuberculosis in Ghana. Trop Med Int Health 1997; 2: 978-981.

7 Lawn SD, Kerkhoff AD, Vogt M, Wood R. HIV-associated tuberculosis: relationship between disease severity and the sensitivity of new sputumbased and urine-based diagnostics. BMC Med 2013; 11: 231.

8 Lawn S D, Kerkhoff A D, Vogt M, Wood R. Clinical significance of lipoarabinomannan detection in urine using a low-cost point-of-care diagnostic assay for HIV-associated tuberculosis. AIDS 2012; 26: 1625-1643.

9 Gupta-Wright A, Fielding K L, van Oosterhout J J, et al. Rapid urinebased screening for tuberculosis to reduce AIDS-related mortality in hospitalized patients in Africa (the STAMP trial): study protocol for a randomised controlled trial. BMC Infect Dis. 2016; 16: 501.

10 Lawn S D, Mwaba P, Bates M, et al. Advances in tuberculosis diagnostics: the Xpert MTB/RIF assay and future prospects for a point-of-care test. Lancet Infect Dis 2013; 13: 349-361.

11 Harries A D, Zachariah R, Corbett E L, et al. The HIV-associated tuberculosis epidemic - when will we act? Lancet 2010; 375: 19061919. 
12 Lawn S D. Africa-not lost but still sick. Implementing interventions to prevent HIV-associated tuberculosis. Int J Tuberc Lung Dis 2014; 18: 1135-1136.

13 Lawn S D, Wood R. Tuberculosis control in South Africa - Will HAART help? S Afr Med J 2006; 96: 502-504.
14 Lawn S D, Harries A D, Williams B G, et al. Antiretroviral therapy and the control of HIV-associated tuberculosis. Will ART do it? Int J Tuberc Lung Dis 2001; 15: 571-581.

15 Lawn S D. Preventing HIV-associated tuberculosis with antiretroviral therapy: shut the stable door early! Int J Tuberc Lung Dis 2015; 19: 3-4. 